\title{
Quasielastic Neutron Scattering Study on the Effect of Blending on the Dynamics of Head-to-Head Poly(propylene) and Poly(ethylene-propylene)
}

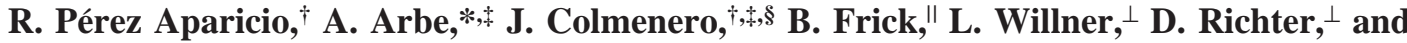 \\ L. J. Fetters" \\ Departamento de Física de Materiales, UPV/EHU, Apartado 1072, 20080 San Sebastián, Spain; \\ Unidad Física de Materiales (CSIC-UPV/EHU), Apartado 1072, 20080 San Sebastián, Spain; \\ Donostia International Physics Center, Paseo Manuel de Lardizabal 4, 20018 San Sebastián, Spain; \\ Institut Laue-Langevin, BP 156, 38042 Grenoble Cedex 9, France; Institut für Festkörperforschung, \\ Forschungszentrum Jülich GmbH, D-52425 Jülich, Germany; and School of Chemical and \\ Biomolecular Engineering, Cornell University, Ithaca, New York 14853
}

Received September 15, 2005; Revised Manuscript Received November 29, 2005

\begin{abstract}
By means of quasielastic neutron scattering, we have investigated the dynamics of two polymers, head-to-head poly(propylene) (HHPP) and poly(ethylene-propylene) (PEP), through the incoherent scattering function of hydrogens, i.e., the $\mathrm{H}$ self-correlation function. Backscattering techniques have allowed us to cover mesoscopic time scales in the momentum transfer region $0.2 \leq Q \leq 1.8 \AA^{-1}$. For both polymers, the glassy dynamics below the glass-transition temperature $T_{\mathrm{g}}$ (HHPP: $T_{\mathrm{g}}=248 \mathrm{~K}$; PEP: $T_{\mathrm{g}}=213 \mathrm{~K}$ ) is dominated by the methyl group rotations. In the temperature region investigated in the supercooled liquid state-well above the glass transition-we have observed a qualitatively similar behavior for both systems: anomalous sublinear diffusion with deviations from Gaussian behavior in the high- $Q$ range. Because of the differences in $T_{\mathrm{g}}$, the time scales associated with HHPP are slower than those observed for PEP in this regime. These results have been taken as reference to address the question of the dynamic miscibility in the blend system composed by a mixture of 50\% HHPP/50\% PEP. Exploiting isotopic labeling, we could experimentally isolate the HHPP-component dynamics in the blend by using a mixture of protonated HHPP and deuterated PEP. In the glassy state, we found that the methyl group dynamics of HHPP is not affected by blending. On the other hand, well above the average glass transition of the blend, the hydrogen motions in HHPP become faster in the presence of PEP. The combined analysis of these results and measurements on the fully protonated blend allowed us finally to deduce also the PEP-component dynamics in the blend in the supercooled liquid state. This dynamics is slowed down by blending but remains faster than that shown by the HHPP component in the blend. These results are discussed in the light of a recent model for blend dynamics proposed by Lodge and McLeish based on the concept of self-concentration. The experimentally observed behavior is well predicted by such a model in the $Q$ and temperature range investigated well above the glass transitions of the polymers.
\end{abstract}

\section{Introduction}

The complexity of the dynamics of glass-forming systems, and, in particular, of glass-forming polymers, has been largely evidenced during past years. In polymers we can identify vibrations, localized motions as methyl group rotations, secondary relaxations, the structural relaxation and large-scale dynamics such as the entropy-driven Rouse motion, reptation, and finally the diffusion of the whole chain. The length scales as well as the characteristic time scales associated with these processes can increase from some tenths of angstroms to hundreds of nanometers and from tenths of picoseconds to years. It is clear that insight at a molecular level is imperative in order to fully characterize and understand the different processes. Neutron scattering (NS) offers space/time resolution on a molecular level and has a great potential to yield such an insight. During past years an important effort has been made in this direction. Particular emphasis has been directed toward a determination of the atomic motions in the $\alpha$-relaxation regime.

\footnotetext{
$\dagger \mathrm{UPV} / \mathrm{EHU}$

$\doteqdot \mathrm{CSIC}-\mathrm{UPV} / \mathrm{EHU}$.

$\S$ Donostia International Physics Center.

"Institut Laue-Langevin.

$\perp$ Forschungszentrum Jülich $\mathrm{GmbH}$.

\# Cornell University.
}

However, features such as the much discussed characteristic length scale associated with this process have not been unveiled so far. The understanding of the vitrification phenomenon demands, in parallel with theoretical development, the compilation of a maximum range of experimental investigations on the dynamical processes characteristic of glass-forming systems, especially at a molecular level.

Recently the question of the dynamical miscibility in thermodynamically miscible polymer blends has attracted increasing interest (see, for instance, the works ${ }^{1-7}$ and references therein). This can be formulated as: how is a given dynamical process affected by blending? Can we distinguish each blend component from a dynamical point of view, or do they move similarly when blended? It is noteworthy that, in addition to the technological importance of these systems, the investigation of the dynamical miscibility could also shed some light on the problem of the length scales associated with the different dynamical processes in polymers, and in particular to the $\alpha$-relaxation, ${ }^{3}$ thereby contributing to the general understanding of the glass transition. From an experimental point of view, the problem of dynamic miscibility in polymer blends requires the utilization of selective techniques to follow the dynamical behavior of the components separately. Until now, most of such studies have been carried out by relaxation techniques like dielectric spectroscopy (DS) 
and NMR (see, e.g., refs 8-17). These techniques are-or can be-selective (DS in the case of a large difference in the dipole moment of the components). However, they do not provide direct spatial information. Only a few studies have taken advantage of the potential offered by NS techniques based on the selectivity which is achieved by deuteration labeling. To date, the only blend systems investigated by NS are poly(vinyl methyl ether)/polystyrene (PVME/PS), ${ }^{9,18}$ polyisoprene/poly(vinylethylene) (PI/PVE), ${ }^{19-21}$ poly(ethylene oxide)/poly(methyl methacrylate) (PEO/PMMA), ${ }^{22,23}$ and poly(dimethylsiloxane)/ poly(ethylmethylsiloxane) (PDMS/PEMS). ${ }^{24}$ The phenomenology emerging by now as a result of the different experimental approaches points to the following scenario: blending does not appreciably affect fast motions and processes active in the glassy state-and consequently are of rather localized character-like e.g. methyl group rotations (NS on $\mathrm{PVME} / \mathrm{PS}^{18}$ ) or even secondary relaxations (DS on $\mathrm{PVME}^{\mathrm{PS}}{ }^{9}$ and PEO/PMMA ${ }^{10}$ and NS on PI/PVE ${ }^{19}$ ). However, segmental and chain dynamics are strongly influenced by the presence of other chains (see, e.g., refs 9, 21, and 25-31 using DS, NMR, or NS). Concerning the $\alpha$-relaxation, two main effects of blending have been well established until now. If we observe selectively the dynamic response of one of the two components, we realize that there is a symmetric broadening of any relaxation function with respect to the homopolymer behavior. This broadening dramatically increases as the temperature decreases down to the average glass transition temperature of the blend. On the other hand, usually two different mean relaxation times are observed, each of them corresponding to the dynamics of each of the components modified by blending. This is what is called "dynamic heterogeneity". From a theoretical point of view, two kinds of approaches have been proposed to account for such observations: one is based on the idea that concentration fluctuations are responsible for both the broadening and the heterogeneity of the dynamics ${ }^{32-34}$ and a second one where the effect of chain connectivity on the local effective concentration of polymer blends (the so-called self-concentration effect) is considered as the main ingredient. ${ }^{1,26}$

In this work we have tried to contribute to the general characterization of the dynamical processes in glass-forming polymers at a molecular level and to the problem of the dynamical miscibility. Thereby, both the glassy and the supercooled liquid states were studied by NS addressing the hydrogen dynamics in two polymers, head-to-head polypropylene (HHPP) and poly(ethylene-propylene) (PEP), as homopolymers and in a blend $(50 \% / 50 \%)$. This has been possible by exploiting deuteration labeling. The two polymers exhibit significantly different glass-transition temperatures $T_{\mathrm{g}}\left(T_{\mathrm{g}}{ }^{\mathrm{HHPP}}=248 \mathrm{~K}, T_{\mathrm{g}}{ }^{\mathrm{PEP}}\right.$ $=213 \mathrm{~K}$ ), giving rise to important differences in their dynamics at high temperatures. We have used the backscattering technique, covering times of the order of the nanosecond and length scales of the order of angstroms. Therefore, we have focused our investigation on temperatures well above the glass transitionthe range where the dynamics of the $\alpha$-relaxation is centered in the experimental NS window. At such high temperatures nonequilibrium effects recently observed in blends below the average $T_{\mathrm{g}}{ }^{23,35}$ are not expected. Moreover, though the effect on the dynamics of thermally driven concentration fluctuations does not completely dissappear at these temperatures, its importance is smaller, and only the self-concentration effects are expected to become evident.

\section{Experimental Section}

A. Sample. Head-to-head polypropylene and alternating poly(ethylene-propylene) were prepared via saturation of the precursor polydienes poly(2,3-dimethylbutadiene) and polyisoprene. The polydienes were obtained via standard anionic polymerization techniques ${ }^{36,37}$ using $s e c$-butyllithium as initiator and benzene as polymerization solvent. The saturation with hydrogen or deuterium was done with a conventional palladium on barium sulfate catalyst. The polydispersity of the polymers, $M_{\mathrm{w}} / M_{\mathrm{n}}$, was smaller than 1.05 as determined by size exclusion chromatography (SEC). Absolute molecular weights were obtained by SEC combined with a triple detector array from Viscotek, model 300: HHPP, $M_{\mathrm{w}}=28.5 \mathrm{~kg} /$ mol; h-PEP, $M_{\mathrm{w}}=20 \mathrm{~kg} / \mathrm{mol}$; d-PEP, $M_{\mathrm{w}}=22 \mathrm{~kg} / \mathrm{mol}$. Blends ${ }^{38,39}$ (50\% HHPP/50\% PEP) were prepared by dissolving the polymers in toluene. To obtain the specimens for NS experiments toluene solutions were cast into aluminum cells. The glass transition temperatures were determined by DSC: $213 \mathrm{~K}$ (PEP), $248 \mathrm{~K}$ (HHPP), and $228 \mathrm{~K}$ (blend).

B. Neutron Scattering Experiments. Neutron scattering experiments reveal structure and dynamics of the sample investigated. ${ }^{40-43}$ This information is obtained by analyzing the change in energy $(\hbar \omega)$ experienced by neutrons scattered into a a given solid angle between $\Omega$ and $\Omega+\mathrm{d} \Omega$. The modulus of the momentum transfer $Q$ is determined by the scattering angle $\theta_{\mathrm{s}}$ and the wavelength of the incoming neutrons $\lambda$ as $Q=4 \pi \sin \left(\theta_{\mathrm{s}} / 2\right) / \lambda$. The measured intensity as a function of $Q$ and energy transfer $\hbar \omega$ contains several contributions:

$$
I(Q, \omega)=\sum_{\alpha} n_{\alpha} \sigma_{\mathrm{inc}}^{\alpha} S_{\mathrm{inc}}^{\alpha}(Q, \omega)+4 \pi\left(\frac{\partial \sigma}{\partial \Omega}\right) \tilde{S}_{\mathrm{coh}}(Q, \omega)
$$

Let us first focus on the incoherent contribution. Here, $n_{\alpha}$ is the relative fraction of nuclei of kind $\alpha$ in the sample. The sum runs over all possible kinds of atoms. $\sigma_{\text {inc }}^{\alpha}$ and $S_{\text {inc }}^{\alpha}(Q, \omega)$ are the incoherent cross section and incoherent scattering function for atoms of type $\alpha$, respectively. $S_{\text {inc }}^{\alpha}(Q, \omega)$ is the Fourier transform of the intermediate incoherent scattering function $S_{\text {inc }}^{\alpha}(Q, t)$, and the double Fourier transform of $S_{\text {inc }}^{\alpha}(Q, \omega)$ yields the self-part of the Van Hove correlation function, $G_{\text {self }}^{\alpha}(r, t)$. In the classical limit, $G_{\text {self }}^{\alpha}(r, t)$ is the probability of a given nucleus of kind $\alpha$ to be at distance $r$ from the position where it was located at a time $t$ before. Thus, incoherent scattering looks at correlations between the positions of the same nucleus at different times. We note that $\sigma_{\text {inc }}^{\alpha}$ changes from isotope to isotope $\left(\sigma_{\text {inc }}^{\mathrm{H}}=80.27\right.$ barns; $\sigma_{\text {inc }}^{\mathrm{D}}=2.05$ barns; $\sigma_{\text {inc }}^{\mathrm{C}}=0$ ). Moreover, given a specific isotope, not all such atoms have necessarily to move in the same way. For example, hydrogens in a methyl group show a richer dynamics than those linked to the main chain because they can, in addition to the segmental motion, participate in the methyl group rotation. Therefore, the label $\alpha$ may refer to the type of isotope (hydrogen, deuterium, carbon, ...) or to a subgroup of the same isotopes (e.g., methyl group hydrogens). The total incoherent cross section is $\sigma_{\text {inc }}=\sum_{\alpha} n_{\alpha} \sigma_{\text {inc }}^{\alpha}$.

The coherent part in eq 1 deals with relative positions of atomic pairs, i.e., collective dynamics. Its intensity is determined by the differential scattering cross section $(\partial \sigma / \partial \Omega)_{\mathrm{coh}}=\sigma_{\mathrm{coh}} S(Q) /(4 \pi)$. This means that it reveals directly the partial structure factor $S(Q)$ (which limit is 1 for $Q \rightarrow \infty$ ) and is weighted by the coherent cross section $\sigma_{\text {coh }}=\sum_{\tilde{\alpha}} n_{\alpha} \sigma_{\text {coh }}^{\alpha}\left(\sigma_{\text {coh }}^{\mathrm{H}}=1.76\right.$ barns, $\sigma_{\text {coh }}^{\mathrm{D}}=5.59$ barns, $\sigma_{\text {coh }}^{\mathrm{C}}=5.55$ barns). $\tilde{S}_{\text {coh }}(Q, \omega)$ is the normalized coherent scattering function (area $=1$ ) that carries the dynamic information: it is determined by the temporal evolution of the atomic pair correlations.

We note that, as NS spectrometers offer a limited energy resolution, the measured functions are affected by the normalized instrumental resolution function, $R(Q, \omega) . R(Q, \omega)$ is the obtained spectrum when purely elastic $(\hbar \omega=0)$ scattering events take place in the sample [i.e., it is the "image" of $\delta(\omega)$ ]. It can usually be determined from the scattering of the sample at very low temperature, where all the dynamical processes are frozen. Thus, the experimentally accessed quantity has to be compared with the convolution of the model function and the resolution, $I_{\exp } \sim I(Q, \omega)$ $\otimes R(Q, \omega)$ 
1. Backscattering Measurements. In a backscattering experiment, perfect crystals are used as monochromator and analyzers, and the instrumental resolution is optimized by using backscattering geometry at both crystals. ${ }^{44}$ These spectrometers work under inverse geometry condition; i.e., the energy of the detected neutrons is fixed to a given value $E_{\mathrm{f}}$ while the energy of the incident neutrons is varied around $E_{\mathrm{f}}$.

In this work we used the backscattering instrument IN16 at the ILL $^{45}$ where the monochromator and the analyzers are made of perfect $\mathrm{Si}(111)$ single crystals. The energy variation is performed by moving the monochromator and exploiting the Doppler effect. In this configuration the wavelength used is $\lambda=6.271 \AA$, the energy window of the experiment is limited to $-15 \mu \mathrm{eV} \leq \hbar \omega \leq$ $15 \mu \mathrm{eV}$ at an energy resolution of $\delta E(\mathrm{hwhm}) \approx 0.4 \mu \mathrm{eV}$, and with 19 different detectors the $Q$ range covered was between 0.19 and $1.81 \AA^{-1}$.

The two fully protonated homopolymers and the partially deuterated blend were investigated in the glassy state at $T=120$, $140,160,180$, and $200 \mathrm{~K}$ (measuring times of about $3 \mathrm{~h}$ ) and in the supercooled liquid state at $300,325,350,375$, and $400 \mathrm{~K}$ (measurements of $\approx 10 \mathrm{~h}$ ). The fully protonated blend was studied at $300,325,350$, and $400 \mathrm{~K}$, with measuring times of $\approx 7 \mathrm{~h}$. The flat-shaped samples were positioned at $135^{\circ}$ with respect to the incident beam. The thicknesses were such that the expected value of the transmission was $90 \%$ in all cases. The experimental resolution function was obtained from the measurement of each sample at $2 \mathrm{~K}$, and the efficiency of the detectors was determined by a Vanadium measurement. Background corrections were performed for the scattering of the empty cell, which was subtracted with the proper transmission factor.

C. Contributions to the Measured Scattering. In the case of protonated polymers, the scattered intensity is generally dominated by the incoherent contributions from the hydrogens in the sample. This is due to the high value of $\sigma_{\text {inc }}^{\mathrm{H}}$ as compared to the other scattering cross sections. Thus, for the three fully protonated samples investigated in this work, the scattered intensity reveals predominantly the self-motions of their hydrogens ( $\sigma_{\text {inc }}$ amounts to $95 \%$ of the total scattering). An essential element of NS dealing with soft matter problems is the huge difference between the cross sections of hydrogen and deuterium, which allows masking the contribution of some molecular groups or components of the system and following the isolated signal of hydrogens in the sample. For this reason, to selectively investigate the dynamics of one component in a blend, mixtures of protonated and deuterated chains are used. In this work we exploited this advantage for isolating the HHPP dynamics in the blend by measuring the scattering of a blend where the PEP component was fully deuterated, HHPP/dPEP. The signal from this sample is dominated by the incoherent contribution from the HHPP hydrogens $\left(\sigma_{\mathrm{coh}} / \sigma_{\text {inc }}=0.15\right)$. Finally, as unfortunately no deuterated HHPP was available to isolate the PEP component, we measured the scattering of a fully protonated blend, HHPP/ PEP. There, the incoherent contributions corresponding to the hydrogens of the two polymers are almost equally weighted $\left(n_{\mathrm{H}, \mathrm{HHPP}} / n_{\mathrm{H}, \mathrm{PEP}}=1.03\right)$.

To experimentally prove that the scattering of our blend samples is predominantly incoherent, we performed neutron diffraction measurements with polarization analysis ${ }^{43}$ using the DNS instrument at the Forschungszentrum Jülich. This technique allows to separate the coherent and incoherent contributions $(\partial \sigma / \partial \Omega)_{\text {coh }}$ and $(\partial \sigma / \partial \Omega)_{\text {inc }}$. In the ideal case, the latter should be $Q$-independent and given by $\sigma_{\text {inc }} / 4 \pi$. The results obtained for both blend samples are shown in Figure $1 .{ }^{46}$ As can be seen, in the $Q$ range of interest in this work the signal is dominated by the incoherent scattering even for the partially deuterated blend (note that in the peak at $\approx 1.2 \AA^{-1}$ the coherent intensity is less than $25 \%$ of the total signal). The strong increase of the scattering at low $Q$ in this sample is due to the coherent contribution produced by the contrast between the labeled macromolecules and the matrix. This scattering reveals the form factor of the chains.

Taking into account these considerations, we can approximate to a large extent $I(Q, \omega) \approx \sigma_{\text {inc }}^{\mathrm{H}} S_{\text {inc }}^{\mathrm{H}}(Q, \omega)$, with $S_{\text {inc }}^{\mathrm{H}}(Q, \omega)=$

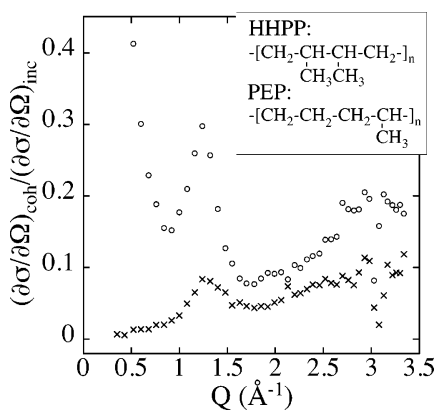

Figure 1. Ratio between the coherent and incoherent intensities scattered by the two blends investigated, as obtained from DNS at 300 $\mathrm{K}$ : empty circles correspond to the sample where the PEP component is deuterated (HHPP/dPEP) and crosses to the fully protonated sample (HHPP/PEP). The inset shows the chemical formulas for the two protonated homopolymers.

$\sum_{H_{i}} n_{\mathrm{Hi}} S_{\mathrm{inc}}^{\mathrm{H}_{\mathrm{i}}}(Q, \omega)$ in case the dynamics of different hydrogens $\mathrm{H}_{i}$ in the system investigated are different.

\section{Results and Analysis}

A. Dynamics in the Glassy State: Methyl Group Rotations. Both HHPP and PEP contain methyl groups in their structural units (see scheme in Figure 1). Because of the low values of their barriers, the rotations of these groups are usually fast enough to contribute to the backscattering window even below the glass transition temperature. ${ }^{47,48}$ As can be seen in Figure 2, a clear quasielastic broadening is observed for the three samples investigated at $T<T_{\mathrm{g}}$. This quasielastic intensity is weaker for PEP, which contains fewer methyl groups. We can assume that in the glassy state the only active motions in our window are methyl group rotations. For rotational motion with characteristic rate $\Gamma$, the incoherent scattering function can be written as

$$
S_{\text {inc }}^{\Gamma}(Q, \omega)=\operatorname{EISF} \delta(\omega)+(1-E I S F) \frac{1}{\pi} \frac{\Gamma}{\Gamma^{2}+\omega^{2}}
$$

where the elastic incoherent structure factor EISF carries the information about the geometry of the particular motion involved. In the case of $\mathrm{CH}_{3}$ rotation, it is given by

$$
E I S F=\frac{1}{3}\left(1+2 \frac{\sin \left(Q r_{\mathrm{HH}}\right)}{Q r_{\mathrm{HH}}}\right)
$$

where $r_{\mathrm{HH}}=1.78 \AA$ is the distance between the hydrogens in the methyl group. It is well-known that the disorder inherent to the amorphous state leads to distributions of rates in glassy systems. This aspect was introduced by the so-called rotation rate distribution model ${ }^{47-50}$ which considers as a first approximation a $\log -$ normal distribution of hopping rates

$$
H(\log \Gamma)=\frac{1}{\sqrt{2 \pi} \sigma_{\Gamma}} \exp \left[-\frac{\left(\log \Gamma-\log \Gamma_{0}\right)^{2}}{2 \sigma_{\Gamma}^{2}}\right]
$$

$\sigma_{\Gamma}$ is the width of the distribution centered at $\Gamma_{0}$. Thus, the final scattering function for a hydrogen undergoing rotation in a methyl group in a glass forming system, $S_{\text {inc }}^{\text {rot }}(Q, \omega)$, is built by adding the scattering functions of the hydrogens located in the different environments weighted by the distribution function

$$
S_{\text {inc }}^{\text {rot }}(Q, \omega)=\int_{-\infty}^{+\infty} H(\log \Gamma) S_{\text {inc }}^{\Gamma}(Q, \omega) \mathrm{d}(\log \Gamma)
$$




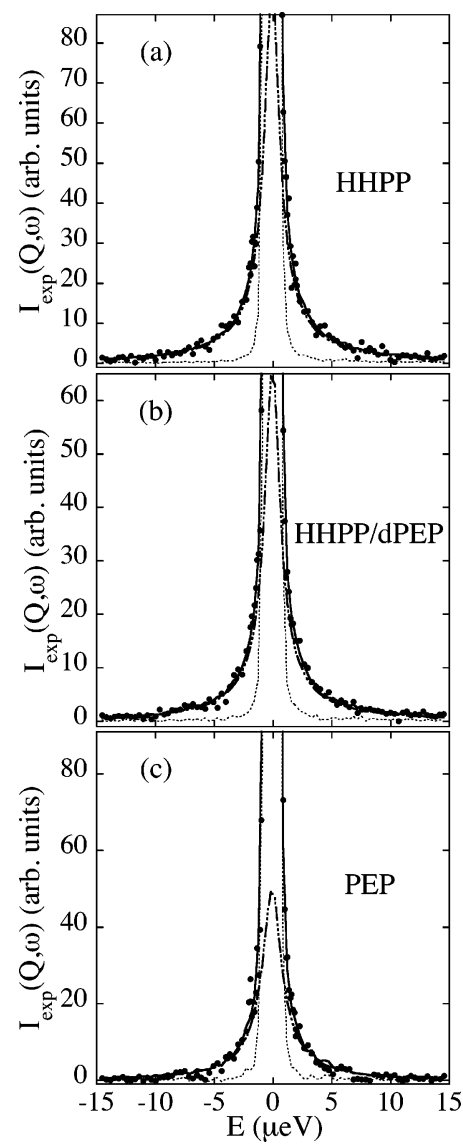

Figure 2. IN16 spectra at $180 \mathrm{~K}$ and $\langle Q\rangle=1.7 \AA^{-1}$ measured from (a) HHPP, (b) HHPP/dPEP, and (c) PEP. Data corresponding to three different detectors $\left(Q=1.64,1.7\right.$, and $\left.1.76 \AA^{-1}\right)$ have been grouped to improve statistics. The solid lines are fits by considering methyl rotations, whose corresponding quasielastic components are depicted by the dashed-dotted lines. The scales are shown at $10 \%$ of the maximum intensities. Dashed lines: instrumental resolution obtained at $2 \mathrm{~K}$.

In addition to the rotational motion, the hydrogens rapidly vibrate around their equilibrium positions. This fast motion leads to an overall decay of the amplitude governed by the so-called Debye-Waller factor $(D W F)$, which directly reveals the meansquared displacement $\left\langle u^{2}\right\rangle$ associated with such fast motions

$$
D W F=\exp \left(-\frac{\left\langle u^{2}\right\rangle}{3} Q^{2}\right)
$$

Assuming $\left\langle u^{2}\right\rangle$ to be the same for all kinds of hydrogens in the sample, the total scattering function for hydrogens can be written as

$$
S_{\mathrm{inc}}^{\mathrm{H}}(Q, \omega)=D W F\left[n_{\mathrm{ch}} \delta(\omega)+n_{\mathrm{MG}} S_{\mathrm{inc}}^{\mathrm{rot}}(Q, \omega)\right]
$$

where $n_{\mathrm{ch}}$ and $n_{\mathrm{MG}}$ are the relative fraction of hydrogens in the main chain and in the methyl groups, respectively. The $\delta(\omega)$ function is the elastic contribution of the main chain protons, which do not participate in the rotations. To account for the small coherent contribution present in the spectra from the HHPP/dPEP sample, following eq 1 we have added an elastic fraction modulated by the corresponding weight as deduced from the DNS measurements (Figure 1). This is justified since motions leading to indistinguishable initial an final atomic configurations do not produce coherent quasielastic scattering. We have assumed that this contribution is affected by the same DWF in a first approximation. Finally, we note that the geometry

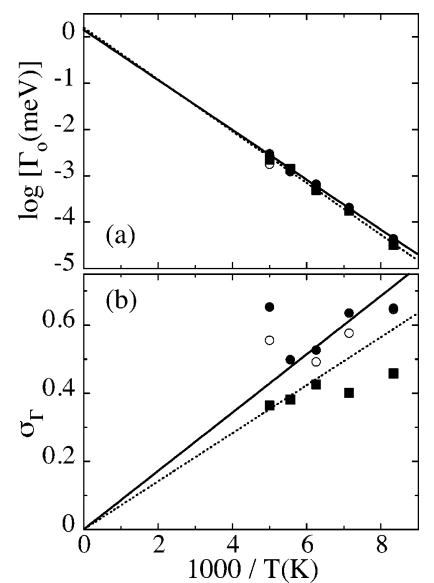

Figure 3. Temperature dependence of the mean value (a) and width (b) of the distributions of rotation rates for methyl groups obtained for HHPP (full circles), PEP (squares), and HHPP in the blend (empty circles). Lines in (a) are Arrhenius fits to the HHPP (solid line) and PEP (dotted line) data; lines in (b) show the expected widths if the distributions are the result of a unique distribution of activation energies of widths $\sigma_{\mathrm{E}}=17 \mathrm{meV}$ (solid line) and $\sigma_{\mathrm{E}}=14 \mathrm{meV}$ (dotted line).

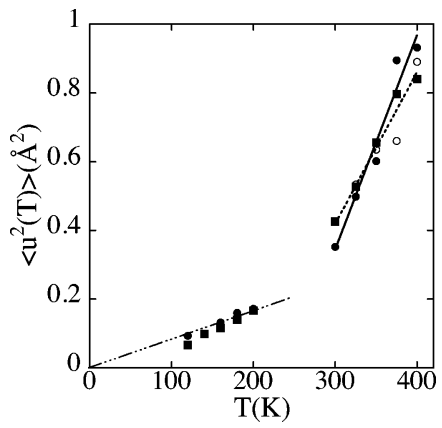

Figure 4. Temperature dependence of the mean-squared displacement associated with the vibrational and fast contributions other than methyl group rotations for HHPP (full circles), PEP (squares), and HHPP in the blend (empty circles). The dashed-dotted line corresponds to a slope of $8.3 \times 10^{-4} \mathrm{~A}^{2} / \mathrm{K}$. The solid and dotted lines show a linear description for the results of HHPP and PEP in the supercooled liquid state, respectively.

of the motion (EISF, eq 3 ) is completely fixed in the model function.

This approach describes the experimental data with high accuracy for the three samples investigated below $T_{\mathrm{g}}$, as can be appreciated in Figure 2. The temperature-dependent values for $\Gamma_{0}$ and $\sigma_{\Gamma}$ resulting from the fits are plotted in Figure 3. Finally, the values of $\left\langle u^{2}\right\rangle$ are displayed as a function of temperature in Figure 4.

B. The Supercooled Liquid State. First we will focus on the results corresponding to the two homopolymers. For the temperature of $350 \mathrm{~K}$, Figure 5 shows in a logarithmic representation their IN16 spectra obtained at three different $Q$ values and normalized to their peak intensities. Figure 6 displays the corresponding curves for $Q=1 \AA^{-1}$ at the lowest and highest temperature investigated. At the same conditions, PEP shows the broadest spectrum and HHPP the narrowest. We remind that the broadening of a spectrum in the quasielastic region is related to the inverse of the characteristic time of the atomic motion leading to such scattering; i.e., the broader the spectrum, the faster is the motion. This means that PEP displays the fastest motion and HHPP the slowest. On the other hand, for a given system, the dynamics reflected in the spectra becomes faster with increasing $Q$ and $T$.

Above the glass transition, the main dynamical process is the structural or $\alpha$-relaxation. Quasielastic incoherent neutron 


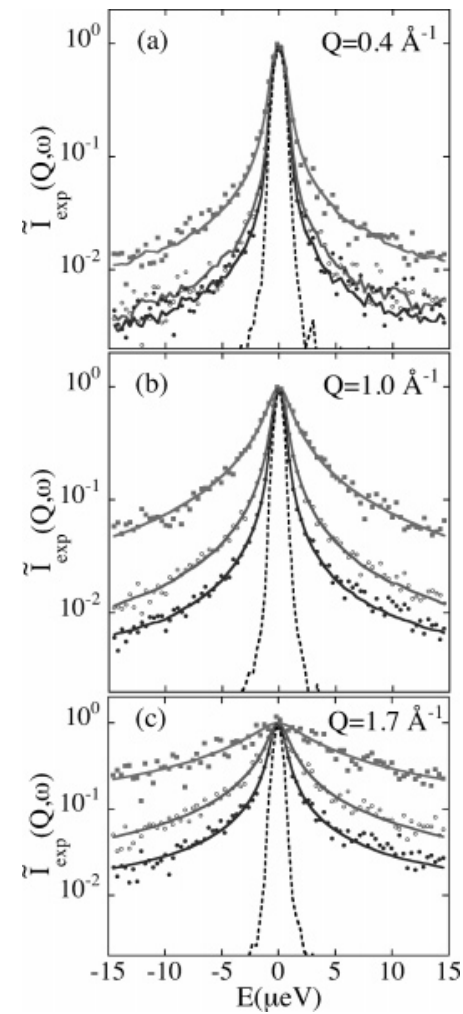

Figure 5. Scattering function normalized to its maximum intensity measured by IN16 for PEP (squares), HHPP (full circles), and the HHPP/dPEP sample (empty circles) at $350 \mathrm{~K}$ and different $Q$ values: $0.4(\mathrm{a}), 1.0$ (b), and $1.7 \AA^{-1}$ (c). The solid lines are fitting curves. The dotted line shows the instrumental resolution.

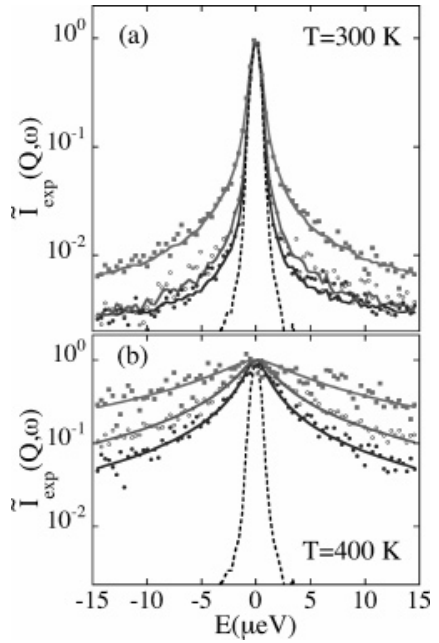

Figure 6. Scattering function normalized to its maximum intensity measured by IN16 for PEP (squares), HHPP (full circles), and the HHPP/dPEP sample (empty circles) at $300 \mathrm{~K}$ (a) and $400 \mathrm{~K}$ (b) for $Q$ $=1.0 \AA^{-1}$. The solid lines are fitting curves. The dotted line shows the instrumental resolution.

scattering investigations ${ }^{51,52}$ and, more recently, molecular dynamics simulations ${ }^{53}$ on glass-forming polymers have shown that the atomic motions in this regime obey a sublinear diffusion; i.e., the mean-squared displacement increases as $\left\langle r^{2}(t)\right\rangle \propto t^{\beta}$ with $\beta<1$. The associated intermediate scattering function can be described by means of a Kohlrausch-Williams-Watts (KWW) or stretched exponential function, so that the scattering function is

$$
S_{\text {inc }}^{\mathrm{KWw}}(Q, \omega)=F T\left\{\exp \left[-\left(\frac{t}{\tau_{\mathrm{w}}}\right)^{\beta}\right]\right\}
$$

The characteristic time $\tau_{\mathrm{w}}(Q, T)$ has been found to follow a power-law $Q$ dependence as $\tau_{\mathrm{w}}(Q, T) \propto Q^{-2 / \beta}$, where $\beta$ is the stretching shape parameter in eq 8 reflecting the deviations of the structure factor from a simple exponential decay. ${ }^{51,52}$ This holds usually in the $Q$ range $0.2-1 \AA^{-1}$ and implies that there the Gaussian approximation is well fulfilled.

In the temperature range investigated, in our dynamical window we expect the observation of the segmental motion involved in the $\alpha$-relaxation. In fact, from the above qualitative considerations, the $Q$ dependence observed for our spectra corresponds well with the scenario of a diffusive-like behavior for the atomic motions above $T_{\mathrm{g}}$. We note that the hydrogens in the methyl groups in principle participate simultaneously in this more global process and in the methyl group rotations. The latter should be very fast at such high temperatures. If these two processes can be considered as statistically independent, as e.g. it was found from MD simulations in polyisoprene, ${ }^{54}$ the total scattering function is built by convolution of the corresponding scattering functions. Considering again the same DWF for all hydrogens, we finally can write the scattering function for hydrogens in one polymer as

$$
\begin{aligned}
S_{\mathrm{inc}}^{\mathrm{H}}(Q, \omega)=D W F\left[n_{\mathrm{ch}} S_{\mathrm{inc}}^{\mathrm{KWw}}\right. & (Q, \omega)+ \\
& \left.n_{\mathrm{MG}} S_{\mathrm{inc}}^{\mathrm{rot}}(Q, \omega) \otimes S_{\mathrm{inc}}^{\mathrm{KWw}}(Q, \omega)\right]
\end{aligned}
$$

This equation can be regrouped into

$$
\begin{aligned}
S_{\mathrm{inc}}^{\mathrm{H}}(Q, \omega)=D W F\left[\left(n_{\mathrm{ch}}+\right.\right. & \left.n_{\mathrm{MG}} E I S F\right) S_{\mathrm{inc}}^{\mathrm{KWW}}(Q, \omega)+ \\
& \left.n_{\mathrm{MG}}(1-E I S F) S_{\mathrm{QE}}^{\mathrm{rot} \& \mathrm{KWW}}(Q, \omega)\right]
\end{aligned}
$$

where $S_{\mathrm{QE}}^{\mathrm{rot} \& \mathrm{KWW}}(Q, \omega)$ is the term giving account for the fast combined rotation and segmental motion of the hydrogens in the methyl group. It is given by

$$
\begin{aligned}
& S_{\mathrm{QE}}^{\mathrm{rot} \& \mathrm{KWW}}(Q, \omega)= \\
& \quad\left[\int_{-\infty}^{+\infty} H(\log \Gamma) \frac{1}{\pi} \frac{\Gamma}{\Gamma^{2}+\omega^{2}} \mathrm{~d}(\log \Gamma)\right] \otimes S_{\mathrm{inc}}^{\mathrm{KWW}}(Q, \omega)
\end{aligned}
$$

This contribution becomes very broad in the temperature range investigated above $T_{\mathrm{g}}$ : for the lowest temperature, $300 \mathrm{~K}$, we can estimate $\Gamma_{0} \approx 21 \mu \mathrm{eV}$ (Figure 3a), i.e., wider than the backscattering window. Therefore, $S_{\mathrm{QE}}^{\mathrm{rot} \& \mathrm{KW}}(Q, \omega)$ will appear practically as a nearly flat background in our window, which intensity decreases with increasing temperature. Thus, we may approximate

$$
S_{\mathrm{inc}}^{\mathrm{H}}(Q, \omega) \approx D W F\left[\left(n_{\mathrm{ch}}+n_{\mathrm{MG}} E I S F\right) S_{\mathrm{inc}}^{\mathrm{KWW}}(Q, \omega)\right]+F B G
$$

We have used this model function to fit the data corresponding to HHPP and PEP. Unfortunately, an accurate determination of the shape parameter $\beta$ by only backscattering measurements is usually not possible due to the convolution with the instrumental resolution involved in the data analysis. Moreover, the presence of a background poses additional difficulties in such determination. Free fits of the highest temperature datawhere, as we will see below, the influence of the background is negligible-lead to values of $\beta=0.47 \pm 0.05$ for HHPP and $\beta=0.53 \pm 0.03$ for PEP. We then chose for both cases a fixed value of 0.5 for the shape parameter $\beta$. This is compatible with our high-temperature analysis and lies well in the range usually found for polymers $(\beta \approx 0.4-0.6){ }^{55}$ As can be appreciated in Figures 5 and 6 , the description of the experimental data is of high quality. From these fits we have obtained the $T$-dependent 
values of $\left\langle u^{2}\right\rangle$ shown in Figure 4 and the characteristic times as a function of $Q$ and $T$ (see Figures 7a and $8 \mathrm{a}$ ). The $Q$ - and $T$-dependent FBG shows a behavior very much compatible with the attributed origin-a DWF(1-EISF)-like modulation with momentum transfer and decreasing values with increasing temperature, such that for $350 \mathrm{~K}$ the $\mathrm{FBG}$ is already almost negligible.

Now let us turn to the results on the blend components. As previously explained, the spectra measured on the partially deuterated sample HHPP/dPEP basically reveal the H-dynamics of the HHPP component. The direct comparison of the NS results on this sample with those of the homopolymers clearly shows that blending affects the dynamics of HHPP hydrogens, leading to faster motions (see Figures 5 and 6). Concerning the shape of the scattering function, it is known ${ }^{9}$ that the extra stretching with respect to the shape of the pure homopolymer becomes negligible at high temperature, where the thermally driven concentration fluctuations seem not to play a very important role anymore. Therefore, we have assumed the same value of 0.5 for the $\beta$ parameter to fit the HHPP/dPEP spectra by means of eq 12. Concerning the coherent contribution of dPEP to the spectra, we note that in this temperature range it cannot be considered as elastic. In fact, as we will see below, the PEP component in the blend is even faster than the HHPP component, and therefore its contribution to the spectra should be quasielastic. Being of coherent nature, some modulation of the characteristic time with the structure factor should be expected for such contribution (see, e.g., ref 55). This means that in the $Q$ range where it shows its maximum intensityclose to $1.2 \AA^{-1}$ (see Figure 1)-this contribution should be relatively slow and therefore rather close to that of HHPP. On the other hand, in the regions where the coherent contribution could be most different from that of HHPP, i.e., close to the minima at $Q \approx 1 \AA^{-1}$ and above $Q \approx 1.5 \AA^{-1}$, this relative coherent intensity amounts to only about $10 \%$ of the measured signal and can be safely neglected. For all these reasons, we have fitted our HHPP/dPEP spectra by considering only one characteristic time scale, that of the HHPP component. Some examples of the good descriptions obtained are shown in Figures 5 and 6 . The resulting values of the fitting parameters are displayed in Figures 4 and $7 \mathrm{~b}$.

With the information about the HHPP component at hand, the effect of blending on the PEP component can be inferred from the experimental results on the fully protonated sample, which contain almost equally weighted contributions from the two polymers. For $Q=1 \AA^{-1}$ and $T=350 \mathrm{~K}$, Figure 9 shows that the HHPP/PEP spectrum is broader than that obtained for HHPP/dPEP, where the PEP component is hidden. Without further analysis, we can immediately deduce that under these conditions the system is dynamically heterogeneous: the PEP component has to move necessarily faster than HHPP in the mixture. Moreover, though at simple sight a quantitative estimate is impossible, the direct comparison with the pure PEP spectrum in Figure 9 points to a slowing down of the dynamics of this polymer in the blend.

The quantitative analysis of the protonated blend was carried out taking into account the contributions of both components:

$$
S_{\text {inc }}^{\mathrm{H}}(Q, \omega)=0.51 S_{\text {inc }}^{\mathrm{H}, \mathrm{HHPP}}(Q, \omega)+0.49 S_{\text {inc }}^{\mathrm{H}, \mathrm{PEP}}(Q, \omega)
$$

where each of the contributions is given by eq 12. From the HHPP/dPEP data analysis we know how to fully characterize the dynamics of HHPP hydrogens in the blend: $\tau_{\mathrm{w}}(Q, T)^{\mathrm{HHPP} / \mathrm{blend}}$ and $\left\langle u^{2}\right\rangle^{\mathrm{HHPP} / \mathrm{blend}}$ can be fixed to the previously obtained values from the HHPP/dPEP data analysis. In fact, we observe that

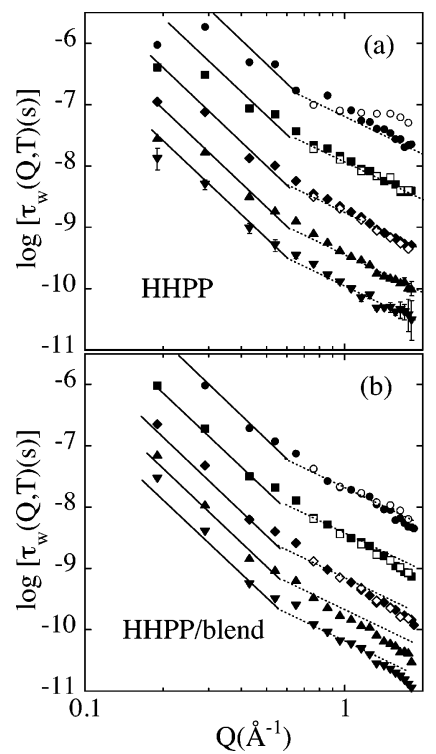

Figure 7. Momentum-transfer dependence of the characteristic times for segmental dynamics obtained for the HHPP hydrogens in the homopolymer (a) and in the blend with HHPP (b) at the different temperatures (in K) investigated: 300 (circles), 325 (squares), 350 (diamonds), 375 (triangles), and 400 (inverted triangles). Full symbols result from the fit with a KWW and a FBG (eq 12). Empty symbols correspond to the fit of eq 9 to the data for selected $Q$ values. The solid and dotted lines display the power laws $\tau_{\mathrm{w}} \propto Q^{-2 / \beta}$ and $\tau_{\mathrm{w}} \propto$ $Q^{-2}$, respectively.

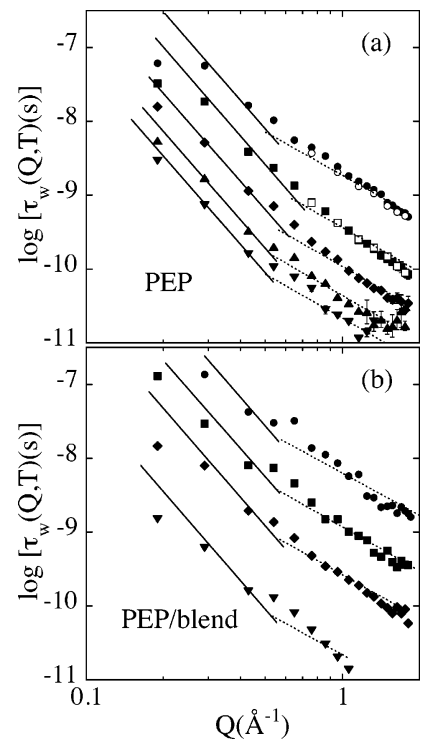

Figure 8. Momentum-transfer dependence of the characteristic times for segmental dynamics obtained for the PEP hydrogens in the homopolymer (a) and in the blend with HHPP (b). Symbols as in Figure 7.

$\left\langle u^{2}\right\rangle^{\mathrm{HHPP} / b l e n d}$ is very close to $\left\langle u^{2}\right\rangle^{\mathrm{PEP}}$ (see Figure 4). Then, it is plausible that $\left\langle u^{2}\right\rangle^{\mathrm{PEP} / b l e n d}$ also coincides with both. This facilitates the analysis, since we can impose more restrictions to our fits. Figure 10 shows the resulting fitting curves with the corresponding contributions (HHPP hydrogens, dashed lines; PEP hydrogens, dashed-dotted lines). We note that, within the uncertainties, the values obtained for the amplitudes of the spectra are well compatible with the hypothesis of similar mean-squared displacements for both components. As expected from the direct comparison of the experimental curves of the blend samples (Figure 9), in the mixture the dynamics of PEP hydrogens is faster than that of HHPP hydrogens. This approach leads to the time scales for the PEP component in the blend 


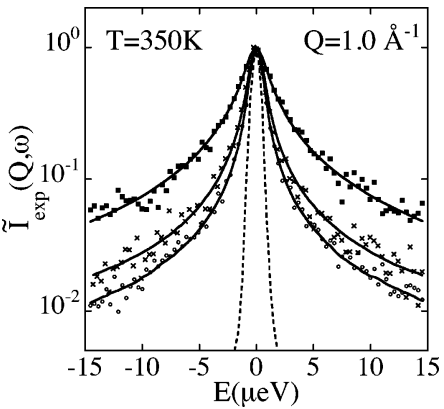

Figure 9. Scattering function normalized to its maximum intensity measured at $350 \mathrm{~K}$ and $Q=1.0 \AA^{-1}$ for PEP (full squares), the fully protonated blend HHPP/PEP sample (crosses), and the partially deuterated blend HHPP/dPEP sample (empty circles). The solid lines are fitting curves. The dotted line shows the instrumental resolution.

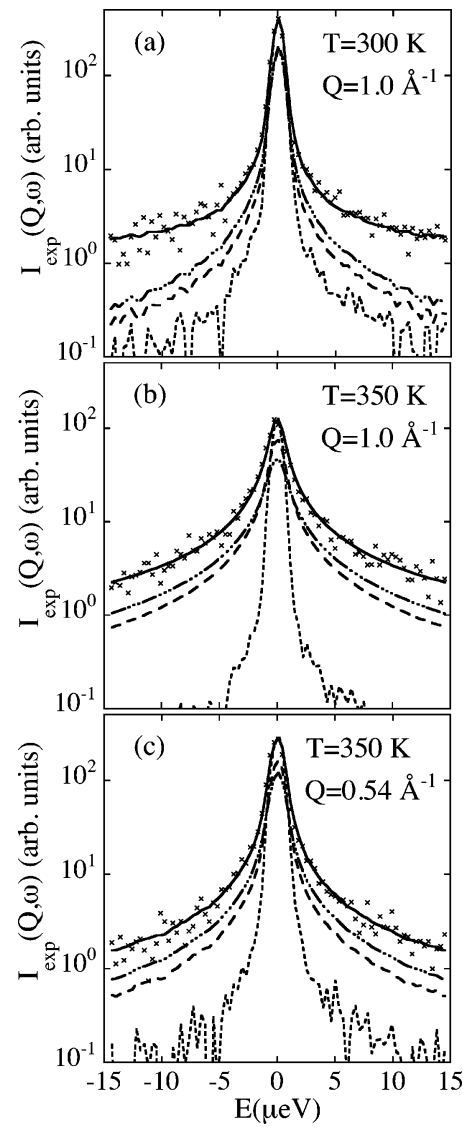

Figure 10. Scattering function of the HHPP/PEP sample for $Q=1.0$ $\AA^{-1}$ at $300 \mathrm{~K}$ (a) and $350 \mathrm{~K}$ (b) and also at $350 \mathrm{~K}$ and $Q=0.54 \AA^{-1}$ (c). The solid lines are fitting curves. Dashed line depicts the HHPP contribution and dashed-dotted the PEP contribution. The dotted line shows the instrumental resolution.

presented in Figure 8b. Finally, Figure 11 compares for two temperatures the time scales obtained for both homopolymers and both blend components as a function of momentum transfer.

\section{Discussion}

A. Sub- $\boldsymbol{T}_{\mathrm{g}}$ Dynamics. Commencing with the two homopolymers, we note that the differences between the values of $\Gamma_{0}$ for both systems are minute (see Figure 3a). The Arrhenius laws

$$
\Gamma_{0}=\Gamma_{\infty} \exp \left[-\frac{E_{0}}{k_{\mathrm{B}} T}\right]
$$

with the preexponential factors $\Gamma_{\infty}^{\mathrm{HHPP}}=1.41 \mathrm{meV}$ and $\Gamma_{\infty}^{\mathrm{PEP}}=$ $1.58 \mathrm{meV}$, and the mean activation energies for the rotation

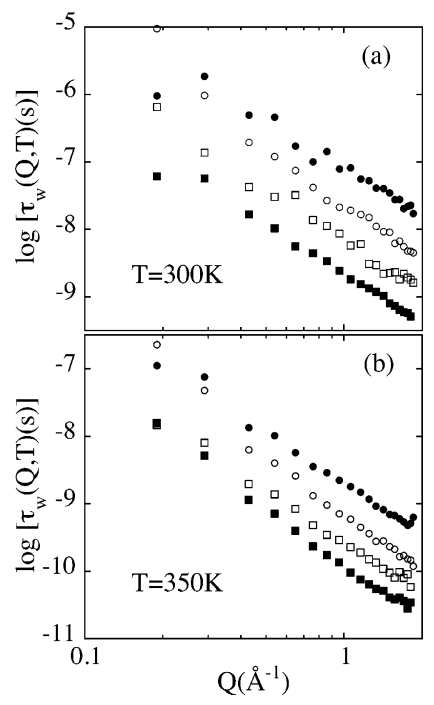

Figure 11. Momentum-transfer dependence of the characteristic times for segmental dynamics obtained for the HHPP hydrogens in the homopolymer (full circles) and in the blend with dPEP (empty circles) and for PEP hydrogens in the homopolymer (full squares) and in the blend with HHPP (empty squares). The temperature in (a) is $300 \mathrm{~K}$ and in (b) $350 \mathrm{~K}$. The crosses show the global time scale obtained when the HHPP/PEP spectra are described by a single KWW. Empty diamonds in (b) show for two selected $Q$ values the time scale obtained for HHPP hydrogens in the blend if the time scale of PEP hydrogens is fixed to that in the pure homopolymer.

$E_{0}^{\mathrm{HHPP}}=107 \mathrm{meV}$ and $E_{0}^{\mathrm{PEP}}=111 \mathrm{meV}$, provide excellent descriptions of these results (see Figure 3a). The distributions, however, are wider in the case of HHPP than for PEP (Figure 3b). If $H(\log \Gamma)$ is a consequence of an underlying Gaussian distribution of activation energies of width $\sigma_{\mathrm{E}}$, the widths are related via $\sigma_{\Gamma}=\sigma_{\mathrm{E}} \log (e) /\left(k_{\mathrm{B}} T\right)$. This implies that $\sigma_{\Gamma}(T)$ continuously decreases, with 0 as limit for $T \rightarrow \infty$. In the case of PEP, the lowest $T$ results for $\sigma_{\Gamma}$ are subjected to high uncertainties, and the data could be well compatible with an energy distribution with $\sigma_{\mathrm{E}} \approx 14 \mathrm{meV}$ (Figure $3 \mathrm{~b}$ ). Somewhat wider $\left(\sigma_{\mathrm{E}} \approx 17 \mathrm{meV}\right)$ would be that for HHPP rotations. The values found for the parameters describing this kind of dynamics are in the same range as those usually found for glass-forming systems. ${ }^{47,48}$ We note that in the case of HHPP a deviation from the expected result from this approach $\left(\sigma_{\Gamma} \propto \sigma_{\mathrm{E}} / T\right)$ is observed at $200 \mathrm{~K}$. This could be related to a change in the distribution due to the vicinity of the glass transition or, as we will discuss later, to the contribution of some secondary relaxational process. It is noteworthy also that for this polymer a librational energy of $28 \mathrm{meV}$ has been reported, ${ }^{56}$ which is in rather good agreement with the expected librational energy of the 3-fold potential governing methyl group rotation in the framework of our results $(26 \mathrm{meV})$. Finally, we have found that the parameters characterizing the methyl group rotations of HHPP in the blend are very close to those in the pure polymer (in fact, many of the corresponding points in Figure 3 are indistinguishable). Even a similar deviation with respect to the line $\sigma_{\mathrm{E}}=17 \mathrm{meV}$ is observed at $200 \mathrm{~K}$ for the broadening of $H(\log \Gamma)$. We can thus conclude that blending does not appreciably affect methyl group rotations, at least for the investigated HHPP component. Similar conclusions were drawn for poly(vinyl methyl ether) methyl group rotations when blended with a higher $T_{\mathrm{g}}$ polymer. ${ }^{18}$

Concerning the DWF, in the range here explored below $T_{\mathrm{g}}$ the two homopolymers show very similar behavior (see Figure 4). The $T$ dependence of the mean-squared displacement in this region can be approximately described by $\left\langle u^{2}\right\rangle\left[\AA^{2}\right] \approx 8.3 \times$ $10^{-4} T[\mathrm{~K}]$ (dashed-dotted line in the figure). In the blend, the 


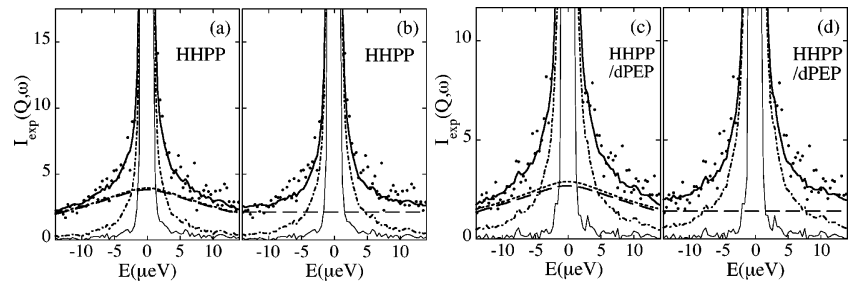

Figure 12. IN16 spectra obtained at $300 \mathrm{~K}$ and $Q=1.64 \AA^{-1}$ for HHPP (a, b) and HHPP/dPEP (c, d). The scale is chosen to show the $4 \%$ of the maximum intensity in both cases. The fitting curves in (a) and (c) (solid lines) have been obtained with the full expression in eq 9, equivalently eq 10 . The dotted lines display the quasielastic contribution to the spectra due to methyl group rotations only. The dashed lines correspond to the convolution of this quasielastic contribution with the $\alpha$-relaxation response. The fitting curves in (b, d) (solid lines) correspond to the approximated eq 12, with the resulting FBG shown as dashed lines. The dashed-dotted lines show in each case the obtained $\alpha$-relaxation function. Thin lines are the instrumental resolution.

determination of $\left\langle u^{2}\right\rangle$ is subjected to uncertainties. These arise from the slight modulation observed for the amplitudes due to the coherent contribution. Since in this temperature range the decay of the amplitudes due to vibrations is rather weak, such modulation prevents a precise determination of $\left\langle u^{2}\right\rangle$. However, we can say that the results of the blend would be compatible with the values obtained for the mean-squared displacements in the homopolymers.

B. Dynamics in the $\alpha$-Relaxation Regime. Following our general scheme, we first center this part of the discussion on the results corresponding to the homopolymers. Let us first focus on the $Q$ dependence of the characteristic times deduced for our systems. As mentioned in the preceding section, the $Q$ dependence expected for the characteristic times $\tau_{\mathrm{w}}(Q, T)$ in the Gaussian approximation is $\tau_{\mathrm{w}}(Q, T) \propto Q^{-2 / \beta}$. For both polymers this law is approximately fulfilled only in the low- $Q$ region (see Figures $7 \mathrm{a}$ and $8 \mathrm{a}$ ). Already for $Q \approx 0.6 \AA^{-1}$ deviations toward a much weaker dependence $\sim Q^{-2}$ can be observed. One could think that these deviations would result partially from the influence of the methyl group rotations on the spectra. However, those were already taken into account allowing for the flat background in the fitting procedure. To check the influence of the approximation done by eq 12, we have used also the full expression (eq 9) to fit the IN16 data. Thereby the extrapolations of the methyl group rotation parameters shown by the lines in Figure 3 have been used. With exception of HHPP at $300 \mathrm{~K}$, indistinguishable results are obtained for the time scales, as can be seen in Figures 7 and 8. Figure 12 shows as representative examples the differences between both approaches for HHPP and HHPP/dPEP, where this backgroud has most relevance: at the lowest temperature investigated and $Q \approx 1.6 \AA^{-1}$, where the weight of the quasielastic contribution due to methyl group rotations, DWF(1-EISF), has a maximum. It is noteworthy that, though broad, the methyl group contribution at this temperature is not completely flat in the IN16 window (Figure 12a,c). The FBG obtained from the fit with eq 12 (Figure 12b,d) matches this contribution at the edges of the IN16 window. However, it underestimates the quasielastic intensity from the methyl groups close to the elastic region. Then, assuming a FBG for methyl group rotations, the remaining observed quasielastic intensity has to be accounted by the $\alpha$-relaxation component. Thus, in the case where the pure-HHPP segmental dynamics is slowest (see Figure 12a,b), this affects the resulting value of $\tau_{\mathrm{w}}$ (see the $300 \mathrm{~K}$ data in Figure $7 \mathrm{a}$ ). However, the fit results are rather insensitive to this approximation when the $\alpha$-relaxation becomes sufficiently fast (for example, like in the blend HHPP/dPEP, as

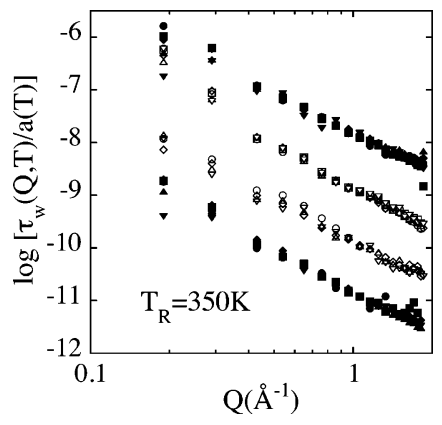

Figure 13. Master curves obtained by superimposing the characteristic times $\tau_{\mathrm{w}}(Q, T)$ at the reference temperature of $350 \mathrm{~K}$ using the appropriate shift factors $a(T)$. Different symbols correspond to different starting temperatures (in K): 300 (circles), 325 (squares), 350 (diamonds), 375 (triangles) and 400 (inverted triangles). Full symbols: homopolymers; empty symbols: blend components. For clarity, the time scales have been multiplied by the following factors: 8 (HHPP), 2 (HHPP in the blend), $1 / 2$ (PEP in the blend), and $1 / 8$ (PEP).

can be seen in Figure 12c,d, and the comparison of the time scales in Figure 7b). In any case, concerning the question of the deviations from Gaussian behavior, Figure 7a shows that the corrections for the lowest temperature lead to even stronger deviations from the power law $Q^{-2 / \beta}$. On the other hand, methyl group dynamics do not affect the higher temperature spectra, where the deviations are also evident. Thus, we find strong nonGaussian dynamics at local scales for both homopolymers in the whole temperature range investigated. Similar deviations from Gaussian behavior in the high $Q$ range have recently been found in other systems. ${ }^{53,57-61}$ Also, in a molecular dynamics (MD) simulation study of HHPP and $\mathrm{PEP}^{62}$ a change in the $Q$ dependence of the time scale for self-motion was found. In that work, however, this occurred at a much higher $Q$ value, $Q \approx$ $1.8 \AA^{-1}$. This might be due to the fact that those simulations used the united atom method, while experimentally the hydrogen dynamics is accessed.

Two possible origins have been proposed to explain such deviations from Gaussian behavior observed at high $Q$. An intrinsic source would be an underlying distribution of discrete jumps leading to the anomalous atomic diffusion in the $\alpha$-relaxation regime. ${ }^{57,60}$ If this is a universal feature, this effect should be present in all glass-forming systems. An additional reason for the deviations would relate to the heterogeneous dynamics of the different kinds of hydrogens (we are in fact assuming the same subdiffusive motion for main chain and methyl group hydrogens). The non-Gaussianity associated with the latter heterogeneity would be superimposed to that originating from the jumplike nature of the atomic subdiffusion, as has been found in the case of poly(vinylethylene). ${ }^{61}$ Unraveling these contributions is only possible by selectively following each kind of atom; unfortunately, this is only possible by fully atomistic MD simulations.

The $Q$ dependences displayed by the characteristic times in Figures $7 \mathrm{a}$ and $8 \mathrm{a}$ hardly depend on temperature, as found in other glass-forming systems. ${ }^{51,63,64}$ This observation allows to build master curves by shifting the time scales corresponding to different temperatures toward the data at a given reference temperature $T_{\mathrm{R}}$. Applying the appropriate shift factors with the intermediate investigated temperature $(350 \mathrm{~K})$ as reference, the master curves shown in Figure 13 have been constructed. The superposition of the data is rather good, indicating that the $Q$ and $T$ dependences of the characteristic times can be factorized to a good approximation or that the $T$ dependence does not depend on the $Q$ value considered. 


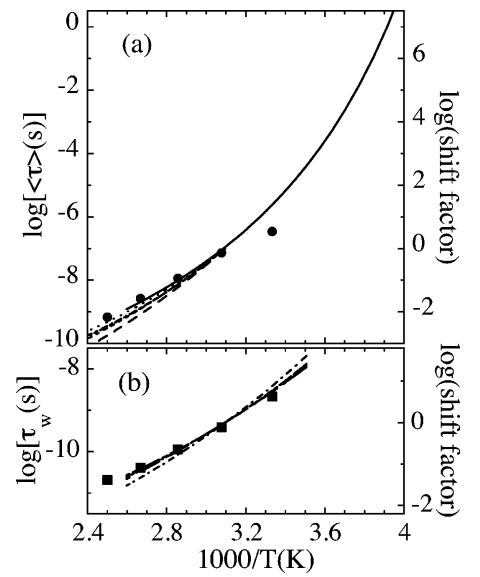

Figure 14. Comparison of the temperature dependence of the characteristic times of the homopolymers observed by NS and by other techniques. In (a), the VF equation reported for HHPP from the combination of NMR and $\mathrm{DS}^{7}$ (solid line) is directly compared with the times obtained from IN16 at $Q=0.65 \AA^{-1}$ (dots). The rheological shift factors reported ${ }^{65}$ for different HHPP samples are also shown (dashed and dotted lines, with the dotted one corresponding to the sample with $T_{\mathrm{g}}=248 \mathrm{~K}$ ). In (b), rheological shift factors obtained for different PEP samples ${ }^{65}$ are compared with the IN16 results at $Q=1$ $\AA^{-1}$ (squares) for PEP.

An interesting question concerning the $\alpha$-relaxation is how do results from different techniques compare. In fact, the combination of information from diverse sources can be very useful due the huge span of the time scales for this process. First, we discuss the case of HHPP. Recently, NMR spin-lattice measurements in the nanosecond region (similar to that accessed by backscattering techniques) have been combined with DS experiments on this polymer, ${ }^{7}$ allowing the determination of the temperature dependence of the segmental motions in the temperature range between $T_{\mathrm{g}}$ and $\approx 390 \mathrm{~K}$. Regarding the shape parameter $\beta$, it was found to increase with increasing temperature from $\approx 0.37$ to $\approx 0.45$ in the interval investigated. It is worth noting that the values obtained by NMR for $\beta$ (0.40.45 ) are in rather good agreement with the value used by us to describe the NS spectra in the same temperature region. On the other hand, a nice overlap of the two sets of data was achieved when the average time $\langle\tau\rangle=\tau_{\mathrm{w}} \Gamma(1 / \beta) / \beta$ for the NMR results was compared with the DS $\tau_{\max }$. The Vogel-Fulcher expression

$$
\tau=\tau_{0} \exp \left[\frac{B}{T-T_{0}}\right]
$$

with $\tau_{0}=11.36 \times 10^{-13} \mathrm{~s}, B=1699.31$, and $T_{0}=198 \mathrm{~K}$, describes simultaneously those data. The direct comparison of those time scales with the NS results is not trivial since the latter depend in addition on the $Q$ value, and slightly different $\beta$ values have been used for the different techniques. We have thus selected the $Q$ value for which the average times obtained by NS coincide best with those reported from NMR. This $Q$ value is $0.65 \AA^{-1}$. As can be seen in Figure 14a, with exception of the $300 \mathrm{~K} \mathrm{NS}$ result, the agreement between the observed $T$ dependences is very good. We also may consider in this comparison the rheological data reported by Gell et al. ${ }^{65}$ on HHPP. With a reference temperature of $323 \mathrm{~K}$, the viscosity shift factors were determined for six samples of different molecular weights and deuteration labels. Though in principle no big differences could be expected, those results show a certain dispersion for the apparent activation energies. These are, in general, higher than that presented by the NS and NMR data. The samples investigated in that work had different $T_{\mathrm{g}}$ values- the reason for that was not known-and seemingly, the rheological data coincide when compared at constant $T-T_{\mathrm{g}}$. We note that for the sample with the same $T_{\mathrm{g}}$ as that here investigated $\left(T_{\mathrm{g}}=248 \mathrm{~K}\right)$ the agreement with the NS and NMR data is quite good (see dotted line in Figure 14a). Therefore, all these results would be finally compatible.

Coming back to the comparison between NMR and NS results, we note that the $Q$ value for which the scattering data match the relaxation times in $\operatorname{HHPP}\left(Q=0.65 \AA^{-1}\right)$ is rather low as compared with the values reported until now $(Q \approx 0.9-$ $\left.1.4 \AA^{-1}\right) .23,53,66$ Those values were obtained from the comparison of NS (or MD simulations) results on the incoherent time for hydrogens with DS or NMR results (either experimental or obtained from MD simulations) for several polymers. Only in the case of poly(vinyl acetate) was a similar $Q$ value reported to match NS and DS data. ${ }^{67}$ The question that arises is whether this $Q$ value could reveal some relevant length scale associated with the relaxation techniques. It is clear that both DS and NMR techniques are probing relatively local length scales (DS through reorientations of the molecular dipoles, NMR through reorientations of bonds). Though a definite interpretation of the associated length scale to the matching $Q$ value is not yet worked out, it was pointed out in ref 53 that this $Q$ value usually lies in the region where the crossover from Gaussian to non-Gaussian behavior is observed in the incoherent scattering function. It is worth noting that again this holds for the case of HHPP. This observation would lead to think that NMR and DS techniques would be somehow sensitive to the heterogeneities and nonGaussian processes causing the deviations from Gaussian behavior. In the framework of an anomalous jump diffusion origin of the $\alpha$-relaxation, ${ }^{57,60}$ these processes could be interpreted as the distributed elementary jumps behind the subdiffusive atomic motions in the segmental relaxation, and these local processes would be the motions revealed by relaxation techniques.

Finally, we comment on the observation at $300 \mathrm{~K}$ of a faster time scale than that expected from combined NMR/DS results. This discrepancy could be due to the difference in the shape parameters used to describe the different sets of data. However, we note that deviations from the temperature dependence predicted by the viscosity have also been observed for the hydrogen self-motions of polyisobutylene ${ }^{58}$ and poly(vinyl chloride). ${ }^{68}$ A similar effect has recently been found for 1,4polybutadiene by fully atomistic MD simulations. ${ }^{69}$ In that work, evidences of local processes were clearly revealed, and the observed decoupling of the time scales was attributed to the contributions from those local processes in addition to the subdiffusive motion in the $\alpha$-relaxation regime. Such a scenario could also be plausible for HHPP. We note that in fact the extra broadening observed for the distribution function of rotational rates at $200 \mathrm{~K}$ for HHPP (Figure $3 \mathrm{~b}$ ) could be an artifact originated from the contribution from localized motions persisting below $T_{\mathrm{g}}$, as it was mentioned in the first part of the discussion. Seemingly, at high enough temperatures the structure factor of hydrogens is dominated by the subdiffusive motion underlying the structural relaxation, while approaching the glass transition other dynamical contributions could affect the scattering function, because polymers are complex systems featuring many internal degrees of freedom. Dielectric measurements in the glassy state are currently being performed by us to characterize the possible secondary relaxations active in these systems. A remaining question could be, if DS and NMR are probing local scales, why do these techniques not reveal also deviations from the VF behavior? In the case of DS, the dynamic 
window at such high temperatures for this techniques only allows following the slowest part of the spectra; i.e., it is sensitive only to the $\alpha$-relaxation process. The NMR experiments, however, could in principle follow the possible additional local processes. The decrease of the $\beta$ parameter with decreasing temperature could be a signature of the occurrence of these motions; as the ratio between average time and KWW time increases with decreasing $\beta$ value, a difference between the time scales as observed by NS and NMR could be found. A discrimination between the two scenarios (subdiffusive process with varying shape or contributions of local processes) could be done with the help of fully atomistic MD simulations.

Concerning the $T$ dependence of the time scales in PEP, a good agreement between rheological and neutron scattering results is found. This can be seen in Figure 14b, where we have compared the NS data a representative $Q$ value $\left(Q=1 \AA^{-1}\right)$ with the shift factors reported for eight different PEP samples. ${ }^{65}$ The average VF parameters for the viscosity measurements are $B=2109.2$ and $T_{0}=145.7 \mathrm{~K}$.

In contrast to the insensitivity upon blending of the sub- $T_{\mathrm{g}}$ dynamics, and as we anticipated from simple inspection of the IN16 spectra, the hydrogen dynamics in the $\alpha$-relaxation regime is accelerated for HHPP when blended with the faster PEP, while PEP slows down in the presence of HHPP chains. This is quantitatively shown in Figure 11. At $300 \mathrm{~K}$, where the time scales of the two homopolymers differ by a factor of about 40 , we find an average difference of a factor of $\approx 4$ between the time scales in the homopolymer melt and in the blend for each system. We note that the shift of the time scales is roughly $Q$-independent, at least at first sight. As the temperature increases, the difference in the time scales of the homopolymer melts diminishes (factor of 18 at $350 \mathrm{~K}$, factor of 6 at $400 \mathrm{~K}$ ), but the behavior found for the blend components remains qualitatively similar (see Figure $11 \mathrm{~b}$ for $350 \mathrm{~K}$ ). Thus, we can conclude that blending induces an acceleration on the high- $T_{\mathrm{g}}$ component and a slowing down of the low- $T_{\mathrm{g}}$ component in this system. We also note again that the blend is dynamically heterogeneous at the length scales experimentally accessible: for a given $Q$ value and temperature, the time scales of both components are clearly different. This kind of behavior has been found in most of the blends investigated up to now: those investigated by relaxation techniques as well as in the few cases studied by quasielastic neutron scattering.

Seeking for evidences of a length scale for dynamic miscibility in this system, we scrutinize the $Q$ dependence of the time scales. The existence of such characteristic length scale should manifest itself in a mutual approach of the relaxation times of different blend components in some $Q$-region, i.e., in a homogenization of the dynamics. However, we observe that the $Q$ dependence is practically the same for all samples (see Figures $7,8,11$, and 13); i.e., the difference in time scales between two given systems is constant for each temperature. Only small differences could be distinguished in detail, as can be seen for HHPP in the blend and as homopolymer. At local scaleshigh- $Q$ region - the acceleration of the dynamics in the blend seems to be more pronounced than at larger length scales. We note, however, that the spectra corresponding to the lowest $Q$ values accessed by IN16 contain a relatively strong contribution of the coherent scattering reflecting the single chain dynamic structure factor (see Figure 1). This scattering is of different nature from that of the fully protonated samples, and therefore the comparison of time scales in this $Q$ range has to be judged with caution. Thus, with the data at hand we find no evidence for the existence of a length scale for miscibility in this system and in the investigated $Q$ range. Neutron spin-echo experiments on the single chain dynamic structure factor of both components in this system are planned for the future. They will address the question of the dynamic miscibility at intermediate and large length scales.

Finally, we discuss our results in the light of the model recently proposed by Lodge and McLeish (LMcL). ${ }^{1}$ Nowadays, it is believed that the dynamics of a segment $i$ of a polymer A in a blend $\mathrm{A} / \mathrm{B}$ is controlled by the local composition of the blend in a volume $V$ around the segment $i$. The LMcL approach assumes that this local composition is determined by the socalled "self-concentration". The idea is that the local concentration of a given blend in a volume $V$ around one segment of one of the blend components will be always richer in this component due to the chain connectivity. The LMcL model considers that, apart from the self-concentration, the composition of volume $V$ is the macroscopic one (i.e., thermodynamic concentration fluctuations are not taken into account). This model also assumes that the relevant volume $V$ is determined by the Kuhn length $b$, defined as ${ }^{70,71}$

$$
b=\frac{C_{\infty} l}{\cos (\theta / 2)}
$$

where $C_{\infty}$ is the characteristic ratio, $l$ the length of the average backbone bond, and $\theta$ the backbone bond angle. The selfconcentration $\phi_{\text {self }}^{\mathrm{A}}$ is determined from the volume fraction occupied by one Kuhn length of polymer A inside a volume $V$ $=b^{3}$

$$
\phi_{\mathrm{self}}^{\mathrm{A}}=\frac{C_{\infty} M_{0}}{k \rho N_{\mathrm{av}} b^{3}}
$$

Here $M_{0}$ is the molar mass and $k$ the number of backbone bonds per repeating unit, and $\rho$ is the density (of course, all these parameters in eq 17, including the characteristic ratio, correspond to polymer A). $N_{\text {av }}$ is Avogadro's number. Finally, the effective local concentration of a given component $\mathrm{A}, \phi_{\mathrm{eff}}^{\mathrm{A}}$, is related with the macroscopic concentration $\phi$ through

$$
\phi_{\text {eff }}^{\mathrm{A}}=\phi_{\text {self }}^{\mathrm{A}}+\left(1-\phi_{\text {self }}^{\mathrm{A}}\right) \phi
$$

The knowledge of the chain dimensions from SANS measurements $^{72}$ allows the calculation of $\phi_{\text {self }}^{\mathrm{HHPP}}$ and $\phi_{\text {self }}^{\mathrm{PEP}}$. At room temperature, the ratios between the unperturbed mean-square end-to-end distance and the molecular weight are $\left\langle R^{2}\right\rangle / M=$ 0.691 and $0.924 \AA^{2} \mathrm{~mol} \mathrm{~g}^{-1}$ for HHPP and PEP, respectively. ${ }^{72}$ Taking into account that for both monomers we find four backbone bonds ${ }^{73}$ of length $l=1.54 \AA$, we obtain $C_{\infty}^{\mathrm{HHPP}}=$ 6.12 and $C_{\infty}^{\mathrm{PEP}}=6.82$. This implies Kuhn lengths of $b^{\mathrm{HHPP}}=$ $11.4 \AA$ and $b^{\mathrm{PEP}}=12.7 \AA$. With $\rho^{\mathrm{HHPP}}=0.878 \mathrm{~g} / \mathrm{cm}^{3}$ and $\rho^{\mathrm{PEP}}$ $=0.856 \mathrm{~g} / \mathrm{cm}^{3},{ }^{72} \phi_{\text {self }}^{\mathrm{HHPP}}=0.17$ and $\phi_{\text {self }}^{\mathrm{PEP}}=0.11$ are deduced at $298 \mathrm{~K}$. These results imply that in our 50\%/50\% system the effective concentrations increase from the average value of 0.5 to values of 0.58 for HHPP and 0.56 for PEP. In the framework of the LMcL model this translates in a shift of the glass transition temperature of each component in the blend with respect to the average or macroscopic glass transition $T_{\mathrm{g}}(\phi)$. The effective local glass transition of component A can be determined from the macroscopic glass transition but evaluated at the corresponding $\phi_{\text {eff. }}^{\mathrm{A}}$. The concentration dependence of the average or macroscopic glass transition of this blend was reported in ref 74 . The set of data provided in that work together with the value obtained 


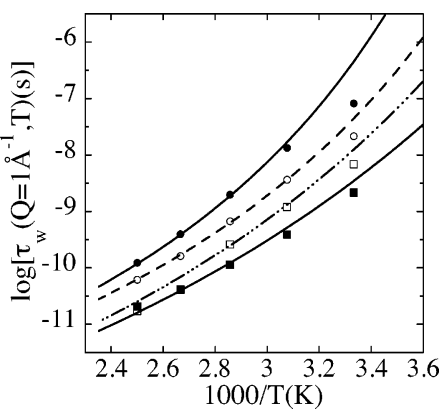

Figure 15. Temperature dependence of the characteristic times corresponding to $Q=1 \AA^{-1}$ obtained for HHPP hydrogens in the homopolymer (full circles) and in the blend with dPEP (empty circles) and PEP hydrogens in the homopolymer (full squares) and in the blend with HHPP (empty squares). The solid lines show the dependence expected from NMR and DS for pure $\mathrm{HHPP}^{7}$ and rheological measurements for pure PEP; ${ }^{65}$ the dashed and dashed-dotted lines are the prediction of the Lodge-McLeish model for the HHPP and PEP blend components, respectively.

by us for our 50\%/50\% sample are best described by the Fox equation

$$
\frac{1}{T_{\mathrm{g}}(\phi)}=\frac{\phi}{T_{\mathrm{g}}^{\mathrm{A}}}+\frac{1-\phi}{T_{\mathrm{g}}^{\mathrm{B}}}
$$

when slightly different $T_{\mathrm{g}}$ values for the homopolymers are used ( $249 \mathrm{~K}$ for HHPP instead of $248 \mathrm{~K}$ and $211 \mathrm{~K}$ for PEP instead of $213 \mathrm{~K})$. The Fox equation provides then $T_{\mathrm{g}, \mathrm{fff}}^{\mathrm{HHPP}}=231.5 \mathrm{~K}$ and $T_{\mathrm{g}, \mathrm{eff}}^{\mathrm{PEP}}=226.2 \mathrm{~K}$. Thus, a difference of about $5 \mathrm{~K}$ in the effective glass transitions of the components is predicted by the model. With respect to the homopolymers, the glass transition of HHPP is reduced in about $16 \mathrm{~K}$, while that of PEP is increased in about $13 \mathrm{~K}^{.75}$ To apply the LMcL model, we have used as starting functions for the $T$ dependence of the segmental motion of the homopolymers the VF parameters reported from NMR and DS for $\operatorname{HHPP}^{7}\left(B=1699.31\right.$ and $T_{0}$ $=198 \mathrm{~K})$ and those deduced from the rheological measurements for PEP $\left(B=2109.2\right.$ and $\left.T_{0}=145.7 \mathrm{~K}\right) .{ }^{65}$ As typical $Q$ value we have chosen $Q=1 \AA^{-1}$. The prefactors corresponding to the KWW times for this $Q$ value are $\tau_{0}=2.6 \times 10^{-14} \mathrm{~s}$ for HHPP and $\tau_{0}=4.0 \times 10^{-15} \mathrm{~s}$ for PEP. Assuming that the parameters $\tau_{0}$ and $B$ of eq 15 are not affected by blending, we have obtained the predicted temperature dependences in the blend, as depicted in Figure 15. As it may be appreciated, the model works well for both components, at least in the high- $T$ range where the VF laws also describe well the thermal evolution of the pure components. At $300 \mathrm{~K}$ a similar deviation from the expected Vogel-Fulcher to that shown by pure HHPP is observed. We could thus conclude that the shift in time scales produced by blending is very well reproduced by the LMcL model for both components. As the shifts observed are independent of the $Q$ value considered in our window, it follows that, within the uncertainties, the model works in the investigated $Q$ range, which corresponds typically to interchain distances. Furthermore, it is worth mentioning that, as expected, the effect of thermally driven concentration fluctuations seems to be very weak at the temperatures investigated. Though a small additional broadening of the spectra with decreasing temperature cannot be discarded-the accuracy in the determination of the shape parameter prevents resolving small spectral-shape changes, mainly where the FBG is more important-the $Q$ dependence of the characteristic times in the blend does not reflect any signature of additional deviations from Gaussian behavior at such low temperatures. Thus, for the system studied, the self- concentration seems to be the dominant factor determining the blend dynamics on the length scales and in the temperature range studied. We note that all temperatures are well above the glass transition of the blend-even above the glass transition of the highest $-T_{\mathrm{g}}$ component - and therefore nonequilibrium effects as those recently observed in other blend systems ${ }^{23,35}$ are not present. We also would like to note that, though we can establish the good agreement with the LMcL model, our data do not provide a very critical check for some of the ingredients of this model. As the experimental data correspond to rather high temperatures, the range of values of $\phi_{\text {self }}$ compatible with the results would be between $\approx 0.08$ and 0.3 for HHPP and between $\approx 0.02$ and 0.3 for PEP. Thus, the question of whether the relevant volume of cooperativity is just that corresponding to the Kuhn length and independent of temperature cannot be definitively answered with our data only.

We finally comment that in a very recent work ${ }^{7}$ on the blend component dynamics of the system HHPP/polyisobutylene (30\%/70\%) the prediction of the LMcL model does not describe the experimentally observed behavior for the HHPP component. A self-concentration of $\approx 0.75$ would be needed for HHPP in order to describe its behavior in the blend (and in a not very satisfactory way). Such a value for $\phi_{\text {self }}$ is not compatible with our data (a maximum value of 0.3 for $\phi_{\text {self }}$ could be estimated to give account for our experimental results on the HHPP component). This situation has been recently rationalized ${ }^{76}$ in terms of a model which combines self-concentration ideas with the Adam and Gibbs model for the $\alpha$-relaxation. In the high temperature range, this approach seems to be compatible with the LMcL model. In any case, it is clear that more experimental work on the widest possible range of systems in parallel with further development of the theoretical frameworks is still demanded in this field. On the other hand, the information provided from MD simulations is also very valuable. Until now, only united atom models have been used to investigate this blend system. ${ }^{77,78}$ In both cases the dynamics of PEP seems to be less affected by blending than the dynamics of HHPP. This result is not in complete agreement with our experimental results. The use of fully atomistic simulations would be a very promising tool to complement the existing sets of experimental and simulated data in this system.

\section{Conclusions}

In this paper we have presented a backscattering study on the hydrogen dynamics of two homopolymers, HHPP and PEP, in the glassy state as well as above their glass-transition temperatures. Below $T_{\mathrm{g}}$, we have fully characterized the methyl group rotations, which reflect the disordered nature of the glass through broad distributions of characteristic times. In the $\alpha$-relaxation regime, we have followed the associated subdiffusive dynamics of hydrogens. Strong deviations from Gaussian behavior are evident for both polymers, which might originate from underlying distributions of elementary jumps leading to the subdiffusive motion, together with dynamical heterogeneities related with the different types of hydrogen in the sample. On the other hand, the temperature dependence dictated by the viscosity is well followed the PEP hydrogen motions in the whole range investigated. This is not the case of HHPP, for which deviations toward a weaker dependence have been observed when approaching the glass transition. These could be a hint for the occurrence of additional localized motions different from methyl group rotations superimposed to the subdiffusive dynamics or a signature of deviations from the time-temperature superposition principle for the $\alpha$-process. The 
identification of the molecular mechanism behind this observation needs further investigations.

The main goal of this work was to investigate the effect of blending on the dynamical processes identified within the homopolymer melts. Therefore, we have performed neutron scattering experiments on isotopic labeled blends in order to study the hydrogen dynamics of each of the components separately in a 50\%/50\% blend. The methyl group rotations in the glassy state are not affected for the HHPP component (the only one investigated below $T_{\mathrm{g}}$ ). In the supercooled liquid state, the high- $T_{\mathrm{g}}$ component (HHPP) is clearly accelerated by blending, while the low- $T_{\mathrm{g}}$ component (PEP) is slowed down. The change in time scales seems to be rather $Q$-independent, at least within the uncertainties. With the data at hand, a determination of a characteristic length of miscibility for this system is not possible, since in the whole $Q$ range investigated the time scales for both components differ by approximately the same factor. Future measurements of the single chain dynamic structure factor of both components will shed some light on this question. Finally, we have found a very good agreement between our results and the prediction of the Lodge and McLeish model. Thus, in this work, we have proven that the effect of the self-concentration is the leading factor determining the dynamical behavior of the blend components in this system, at least in the temperature range well above the glass transition and on spatial scales of the order of the interchain distances.

Acknowledgment. We thank Dr. Werner Schweika and Dr. Madhu Sudan Tyagi and Prof. Angel Alegría for their help at the DNS and IN16 instruments, respectively. This research project has been supported by the European Commission NoE SoftComp, Contract NMP3-CT-2004-502235. R.P., A.A., and J.C. acknowledge support from the projects MAT2004-01017 and 9/UPV00206.215-13568/2001. R.P. acknowledges the grant of "Donostia International Physics Center". The European Commission, NMI3 Contract RII3-CT-2003-505925, supported the experiments at the Forschungsreaktor in Jülich.

\section{References and Notes}

(1) Lodge, T. P.; McLeish, T. C. B. Macromolecules 2000, 33, 5278.

(2) Leroy, E.; Alegría, A.; Colmenero, J. Macromolecules 2003, 36, 7280.

(3) Hirose, Y.; Urakawa, O.; Adachi, K. Macromolecules 2003, 36, 3699.

(4) He, Y.; Lutz, T. R.; Ediger, M. D. J. Chem. Phys. 2003, 119, 9956.

(5) Kant, R.; Kumar, S. K.; Colby, R. H. Macromolecules 2003, 36, 10087.

(6) Colby, R. H.; Lipson, J. E. G. Macromolecules 2005, 38, 4919.

(7) Krygier, E.; Lin, G.; Mendes, J.; Mukandela, G.; Azar, D.; Jones, A. A.; Pathak, J. A.; Colby, R. H.; Kumar, S. K.; Floudas, G.; Krishnamoorti, R.; Faust, R. Macromolecules 2005, 38, 7721.

(8) Alegría, A.; Colmenero, J.; Ngai, K. L.; Roland, C. M. Macromolecules 1994, 27, 4486.

(9) Cendoya, I.; Alegría, A.; Alberdi, J. M.; Colmenero, J.; Grimm, H.; Richter, D.; Frick, B. Macromolecules 1999, 32, 4065.

(10) Dionísio, M.; Fernandes, A. C.; Mano, J. F.; Correia, N. T.; Sousa, R. C. Macromolecules 2000, 33, 1002.

(11) Haley, J. C.; Lodge, T. P.; He, Y.; Ediger, M. D.; von Meerwall, E. D.; Mijovic, J. Macromolecules 2003, 36, 6142.

(12) Lutz, T. R.; He, Y.; Ediger, M. D.; Cao, H.; Lin, G.; Jones, A. A. Macromolecules 2003, 36, 1724.

(13) Lutz, T. R.; He, Y.; Ediger, M. D.; Pitsikalis, M.; Hadjichristidis, N. Macromolecules 2004, 37, 6440.

(14) Cao, H.; Lin, G.; Jones, A. A. J. Polym. Sci., Part B: Polym. Phys. 2005, 43, 2433.

(15) Lutz, T. R.; He, Y.; Ediger, M. D. Macromolecules 2005, 38, 9826.

(16) He, Y.; Lutz, T. R.; Ediger, M. D. Macromolecules 2004, 37, 9889.

(17) He, Y.; Lutz, T. R.; Ediger, M. D.; Pitsikalis, M.; Hadjichristidis, N.; von Meerwall, E. D. Macromolecules 2005, 38, 6216.

(18) Mukhopadhyay, R.; Alegría, A.; Colmenero, J.; Frick, B. J. Non-Cryst. Solids 1998, 235-237, 233.

(19) Arbe, A.; Alegría, A.; Colmenero, J.; Hoffmann, S.; Willner, L.; Richter, D. Macromolecules 1999, 32, 7572.
(20) Doxastakis, M.; Kitsiou, M.; Fytas, G.; Theodorou, D. N.; Hadjichristidis, N.; Meier, G.; Frick, B. J. Chem. Phys. 2000, 112, 8687.

(21) Hoffmann, S.; Willner, L.; Richter, D.; Arbe, A.; Colmenero, J.; Farago, B. Phys. Rev. Lett. 2000, 85, 772.

(22) Sakai, V. G.; Chen, C.; Maranas, J. K.; Chowdhuri, Z. Macromolecules 2004, 37, 9975.

(23) Genix, A.-C.; Arbe, A.; Alvarez, F.; Colmenero, J.; Willner, L.; Richter, D. Phys. Rev. E 2005, 72, 031808.

(24) Götz, H.; Ewen, B.; Maschke, U.; Monkenbusch, M.; Meier, G. e-Polym. 2003, 011.

(25) Colby, R. H. Polymer 1989, 30, 1275.

(26) Chung, G.-C.; Kornfield, J. A.; Smith, S. D. Macromolecules 1994 $27,5729$.

(27) Arendt, B. H.; Kannan, R. M.; Zewail, M.; Kornfield, J. A.; Smith, S. D. Rheol. Acta 1994, 33, 322.

(28) Arendt, B. H.; Krishnamoorti, R.; Kornfield, J. A.; Smith, S. D. Macromolecules 1997, 30, 1127.

(29) Adams, S.; Adolf, D. B. Macromolecules 1999, 32, 3136.

(30) Yang, X.; Halasa, A.; Hsu, W.-L.; Wang, S.-Q. Macromolecules 2001, $34,8532$.

(31) Min, B.; Qiu, X.; Ediger, M. D.; Pitsikalis, M.; Hadjichristidis, N. Macromolecules 2001, 34, 4466.

(32) Zetsche, A.; Fischer, E. W. Acta Polym. 1994, 45, 168.

(33) Katana, G.; Fischer, E. W.; Hack, T.; Abetz, V.; Kremer, F. Macromolecules 1995, 28, 2714.

(34) Kumar, S. K.; Colby, R. H.; Anastasiadis, S. H.; Fytas, G. J. Chem. Phys. 1996, 105, 3777.

(35) Lorthioir, C.; Alegría, A.; Colmenero, Phys. Rev. E 2003, 68, 031805.

(36) Morton, M.; Fetters, L. J. Rubber Chem. Technol. 1975, 48, 359.

(37) Hadjichristidis, N.; Iatrou, H.; Pispas, S.; Pitsikalis, M. J. Polym. Sci., Part A: Polym. Chem. 2000, 38, 3211.

(38) Graessley, W. W.; Krishnamoorti, R.; Reichart, G. C.; Balsara, N. P.; Fetters, L. J.; Lohse, D. J.; Schulz, D. N.; Sissano, J. A. Macromolecules 1994, 27, 2574.

(39) Graessley, W. W.; Krishnamoorti, R.; Reichart, G. C.; Balsara, N. P.; Fetters, L. J.; Lohse, D. J. Macromolecules 1995, 28, 1260

(40) Springer, T. Quasielastic Neutron Scattering for the Investigation of Diffusive Motions in Solids, Liquids; Springer Tracts in Modern Physics; Springer-Verlag: Berlin, 1972; Vol. 64.

(41) Lovesey, S. W. Theory of Neutron Scattering from Condensed Matter; Clarendon Press: Oxford, 1984.

(42) Bée, M. Quasielastic Neutron Scattering; Adam Hilger: Bristol, 1988.

(43) Squires, G. L. Introduction to the Theory of Thermal Neutron Scattering; Dover Publication: New York, 1996.

(44) Alefeld, B.; Birr, M.; Heidemann, A. Naturwissenschaften 1969, 56, 410.

(45) Frick, B.; Gonzalez, M. Physica B 2001, 201, 8.

(46) Pérez Aparicio, R.; Arbe, A.; Colmenero, J.; Schweika, W.; Richter, D. Manuscript in preparation.

(47) Colmenero, J.; Alegría, A. Adv. Funct. Molecules Polym. 2001, 2, 271.

(48) Colmenero, J.; Moreno, A.; Alegría, A. Prog. Polym. Sci., accepted for publication.

(49) Chahid, A.; Alegría, A.; Colmenero, J. Macromolecules 1994, 27, 3282.

(50) Colmenero, J.; Mukhopadhyay, R.; Alegría, A.; Frick, B. Phys. Rev. Lett. 1998, 80, 2350.

(51) Colmenero, J.; Alegría, A.; Arbe, A.; Frick, B. Phys. Rev. Lett. 1992, $69,478$.

(52) Arbe, A.; Colmenero, J.; Monkenbusch, M.; Richter, D. Phys. Rev. Lett. 1998, 81, 590.

(53) Colmenero, J.; Alvarez, F.; Arbe, A. Phys. Rev. E 2002, 65, 041804

(54) Alvarez, F.; Arbe, A.; Colmenero, J. Chem. Phys. 2000, $261,47$.

(55) Richter, D.; Monkenbusch, M.; Arbe, A.; Colmenero, J. Neutron SpinEcho Investigations on Polymer Dynamics; Adv. Polym. Sci. Vol. 174; Springer-Verlag: Berlin, 2005.

(56) Annis, B. K.; Lohse, D. J.; Trouw, F. J. Chem. Phys. 1999, 111, 1699.

(57) Arbe, A.; Colmenero, J.; Alvarez, F.; Monkenbusch, M.; Richter, D.; Farago, B.; Frick, B. Phys. Rev. Lett. 2002, 89, 245701.

(58) Farago, B.; Arbe, A.; Colmenero, J.; Faust, R.; Buchenau, U.; Richter, D. Phys. Rev. E 2002, 65, 051803.

(59) Ahumada, O.; Theodorou, D. N.; Triolo, A.; Arrighi, V.; Karatasos, C.; Ryckaert, J. P. Macromolecules 2002, 35, 7110.

(60) Arbe, A.; Colmenero, J.; Alvarez, F.; Monkenbusch, M.; Richter, D.; Farago, B.; Frick, B. Phys. Rev. E 2003, 67, 051802.

(61) Narros, A.; Alvarez, F.; Arbe, A.; Colmenero, J.; Richter, D.; Farago, B. J. Chem. Phys. 2004, 121, 3282

(62) Neelakantan, A.; Maranas, J. K. J. Chem. Phys. 2004, 120, 465.

(63) Colmenero, J.; Alegría, A.; Alberdi, J. M.; Alvarez, F.; Frick, B. Phys. Rev. B 1991, 44, 7321.

(64) Colmenero, J.; Arbe, A.; Alvarez, F.; Monkenbusch, M.; Richter, D.; Farago, B.; Frick, B. J. Phys.: Condens. Matter 2003, 15, S1127. 
(65) Gell, C. B.; Graessley, W. W.; Fetters, L. J. J. Polym. Sci., Part B: Polym. Phys. 1997, 35, 1933.

(66) Colmenero, J.; Arbe, A.; Alegría, A. J. Non-Cryst. Solids 1994, $172-$ $174,126$.

(67) Tyagi, M.; Alegría, A.; Colmenero, J. J. Chem. Phys. 2005, 122, 244909.

(68) Arbe, A.; Moral, A.; Alegría, A.; Colmenero, J.; Pyckhout-Hintzen, W.; Richter, D.; Farago, B.; Frick, B. J. Chem. Phys. 2002, 117, 1336.

(69) Colmenero, J.; Arbe, A.; Alvarez, F.; Narros, A.; Monkenbusch, M.; Richter, D. Europhys. Lett. 2005, 71, 262.

(70) Boyd, R. H.; Phillips, P. J. The Science of Polymer Molecules; Cambridge University Press: Cambridge, 1993.

(71) Rubinstein, M.; Colby, R. H. Polymer Physics; Oxford University Press: New York, 2003.

(72) Fetters, L. J.; Lohse, D. J.; Richter, D.; Witten, T. A.; Zirkel, A. Macromolecules 1994, 27, 4639.
(73) Because of the presence of some lateral groups, this number is closer to 3.9 in both cases. This small correction does not affect the results.

(74) Gell, C. B.; Krishnamoorti, R.; Kim, E.; Graessley, W. W.; Fetters, L. J. Rheol. Acta 1997, 36, 217.

(75) Sometimes another definition for the Kuhn length is used $\left(l_{\mathrm{K}}=C_{\infty} l\right)$. Then the obtained values for $\phi_{\text {self }}^{\mathrm{HHP}}$ and $\phi_{\text {self }}^{\mathrm{PEP}}$ are 0.29 and 0.20 , respectively, and a difference of $9 \mathrm{~K}$ is obtained for the effective glass transitions. In the temperature range studied in this work, the corresponding Vogel-Fulcher curves are rather similar to those shown in Figure 15.

(76) Cangialosi, D.; Alegría, A.; Colmenero, J. Macromolecules 2005; ASAP.

(77) Luettmer-Strathmann, J. J. Chem. Phys. 2005, 123, 014910.

(78) Neelakantan, A.; May, A.; Maranas, J. K. Macromolecules 2005, 38 , 6598.

MA052006K 